\section{ORIGINAL RESEARCH}

\author{
L. Liauw \\ J. van der Grond \\ A.A. van den \\ Berg-Huysmans \\ L.A.E.M. Laan \\ M.A. van Buchem \\ G. van Wezel-Meijler
}

\title{
Is There a Way to Predict Outcome in (Near) Term Neonates with Hypoxic-Ischemic Encephalopathy Based on MR Imaging?
}

BACKGROUND AND PURPOSE: It has previously been demonstrated that comparison of signal intensity (SI) between selected brain structures on T1-weighted images enables distinction between the absence or presence of hypoxic-ischemic (HI) brain injury in young infants. The aim of the present study was to assess whether this method of brain structure T1-weighted SI comparison also enables prediction of outcome.

MATERIALS AND METHODS: Survivors of a group of 57 children with neonatal HI encephalopathy (HIE) grade 2 or 3 according to Sarnat and Sarnat and controls who underwent neonatal MR imaging were retrospectively assigned to 1 of 3 outcome groups at 5 years of age, depending on developmental outcome: 1) normal, 2) mildly abnormal, and 3) definitely abnormal. Gestational age was not significantly different between the HIE group (range, $35+5-42+5$ weeks; mean, $39+4$ weeks) and control group (range, $35+0-42+1$ weeks; mean, $39+2$ weeks). We calculated the predictive values of the neonatal clinical HIE classification according to Sarnat and Sarnat for outcome (neonatal death and developmental outcome in survivors). We assessed which brain structure T1-weighted SI comparison scored best for outcome prediction. Predictive values of that comparison for outcome were calculated for the entire group and for the HIE grade 2 group only, a patient group with highly variable outcome.

RESULTS: Of the 57 children, 6 died. Outcome group 1 consisted of 31; group 2, of 14; and group 3, of 6 children. The positive predictive value of the neonatal clinical classification for adverse outcome (outcome group 3 and death) was 52\%; and negative predictive value, 100\%. These were respectively $45 \%$ and $0 \%$ in children with HIE grade 2. Of all brain structure T1-weighted SI comparisons, that of the posterior limb of the internal capsule versus the posterolateral putamen scored best for outcome prediction. The positive predictive value for adverse outcome was $69 \%$; and negative predictive value, $98 \%$. In children with HIE grade 2, the positive predictive value and negative predictive value for adverse outcome were $67 \%$ and $88 \%$.

CoNCLUSIONS: Brain structure T1-weighted SI comparisons are helpful to predict outcome in (near) term neonates with HIE. This finding adds to the current knowledge and clinical practice. If the SI in the posterolateral putamen is less than the $\mathrm{SI}$ in the posterior limb of the internal capsule, favorable outcome is very likely, whereas if the SI in the posterolateral putamen is equal to or greater than the $\mathrm{SI}$ in the posterior limb of the internal capsule, adverse outcome is very likely. In neonates with HIE grade 2 according to Sarnat and Sarnat, prediction of outcome is substantially improved by using these brain structure T1-weighted SI comparisons.

$\mathbf{F}$

or neonates with hypoxic-ischemic brain (HI) injury, the predictive value of neonatal MR imaging for neurologic development until school age has been reported. ${ }^{1-4}$ Abnormal or absent myelination of the posterior limb of the internal capsule is associated with impaired motor outcome. ${ }^{1,3,5}$ Abnormal signal intensity (SI) in the basal ganglia and/or the thalami is associated with impaired cognitive and motor outcome. ${ }^{1-5}$ We aimed to assess the predictive values for early neonatal death or developmental outcome at school age of a simple method by using MR imaging without looking at patterns of brain injury.

In a recent article by our group ${ }^{6}$ involving 57 young infants born after a gestational age of $>35$ weeks, we demonstrated that 2 brain structure SI comparisons (the posterior limb of the internal

Received February 13, 2008; accepted after revision May 4.

From the Department of Radiology (L.L., J.v.d.G., A.A.v.d.B.-H., M.A.v.B.), Neurology (L.A.E.M.L.), and Neonatology (G.v.W.-M.), Leiden University Medical Center, Leiden, the Netherlands.

Please address correspondence to L. Liauw, MD, Department of Radiology/667, Radboud University Nijmegen Medical Center, PO Box 9101, 6500 HB Nijmegen, the Netherlands; e-mail: I.liauw@yahoo.com

DOI 10.3174/ajnr.A1188 capsule versus the posterolateral putamen, and the corona radiata versus the peri-Rolandic cortex) on T1-weighted images permitted the prediction of the absence or presence of $\mathrm{HI}$ brain injury and enabled distinction in 37/57 infants between hypoxic-ischemic encephalopathy (HIE) (neonatal HIE grade 2 or 3 according to Sarnat and Sarnat ${ }^{7}$ ) and no HIE. With this method, for each subject, SI scores were assigned to 19 different complete brain structures, on the basis of pair-wise comparisons of SI among the 19 structures. The advantage of this method is that it is easy to implement, by using standard T1-weighted images without a need for advanced MR imaging techniques. The study did not relate MR imaging findings to outcome.

The aim of the present study was to assess whether this method of brain structure T1-weighted SI comparison enables prediction of neonatal death or normal, mildly abnormal, or definitely abnormal developmental outcome at 5 years of age.

\section{Materials and Methods}

\section{Subjects}

The institutional review board approved this study and waived patient informed consent. In the period from January 1990 to 
September 2001, cerebral MR imaging examinations were collected from all infants born after a gestational age of $>35$ weeks who had undergone MR imaging between the first day after birth and $31 / 2$ months (104 days) of age. ${ }^{6}$ Assignment of the 57 subjects ( 35 male) into either the patient or the control group based on the neonatal clinical condition has been described previously. ${ }^{6}$ The patient group consisted of 23 infants ( 16 male) with clinical findings of neonatal HIE compatible with HIE stage 2 lasting at least 5 days $(n=20)$ or HIE stage $3(n=3)$ according to Sarnat and Sarnat. ${ }^{7}$ HIE stage 2 indicates a moderate encephalopathy, with the infant being lethargic and hypotonic and having seizures. In HIE stage 3 , there is a severe encephalopathy, with the infant being comatose and severely hypotonic with decreased or absent reflex activity and a severely depressed electroencephalography (EEG). ${ }^{7}$ The control group consisted of 34 infants (19 male) without a history of perinatal asphyxia or other episodes that might provoke HI cerebral damage and without symptoms of HIE. Of all these children (patients and controls) born after a gestational age of $>35$ weeks, neonatal death was recorded, and in survivors, developmental outcome at school age was retrospectively evaluated. Gestational age was not significantly different between the HIE group and the control group (HIE group: range, $35+5-42+5$ weeks; mean, $39+4$ weeks; control group: range, $35+0-42+1$ weeks; mean, $39+2$ weeks).

The MR images were retrospectively evaluated, and the SI of 19 different brain structures was assessed on T1-weighted images as described previously. ${ }^{6}$ This was done in myelinated structures (medulla oblongata, cerebellar peduncles, pons, mesencephalon, ventrolateral thalamus, rest of the thalamus, globus pallidus, posterolateral putamen, rest of the putamen, head of caudate nucleus, posterior limb of the internal capsule, corona radiata, centrum semiovale, peri-Rolandic cortex, and visual cortex) and unmyelinated structures (peripheral temporal white matter, peripheral occipital white matter, peripheral parietal white matter, and peripheral frontal white matter). The SI of each structure was compared with the SI of all the other 18 structures (pair-wise comparisons).

\section{MR Imaging}

Images were obtained with superconducting magnets (Gyroscan ACS-NT 15; Philips Medical Systems, Best, the Netherlands) operating at a field-strength of 1.5T. T1-weighted spin-echo sequences (TR/ TE, 205-730/4-16 ms), T2-weighted spin-echo sequences (TR/TE, 1553-5897/80-200 ms), and fluid-attenuated inversion recovery sequences (TR/TE/TI, 8000/110-120/1860-2000 ms) were obtained in all infants in the axial plane. In most infants, T1-weighted spin-echo sequences (TR/TE, 205-730/4-16 ms) were also obtained in the sagittal plane. Section thickness ranged from 4 to $7 \mathrm{~mm}$ with an intersection gap of 0.4-0.7 mm. In addition, from 1999 onwards, diffusionweighted images by using single-shot spin-echo echo-planar sequences (TR/TE, 5132-5000/74-68 ms with a b-value of $800-1000$ $\mathrm{s} / \mathrm{mm}^{2}$ ) were routinely obtained in the axial plane.

\section{Follow-up}

Surviving patients and controls visited a pediatric neurologist and/or a pediatrician specializing in neurologic follow-up at regular intervals. A pediatric neurologist (L.A.E.M.L.), blinded to the neonatal clinical data, retrospectively assigned the children at 5 years of age to 1 of the 3 following groups, according to the classification of development by the $\mathrm{WHO}^{8}: 1$ ) "normal" (ie, normal neurologic examination findings and normal cognitive developmental history), 2) "mildly abnormal" (ie, mildly abnormal neurologic findings [mild hypertonia, hypotonia, and/or asymmetry]) and/or mildly abnormal cognitive developmental history (such as mild speech and language delay and mild behavioral disorders and the need for remedial teaching at school), and 3) "definitely abnormal" (ie, children with major neurologic problems, such as cerebral palsy and/or severe cognitive function disorders and/or epilepsia). In most infants, the Van Wiechen examination (a standardized Dutch assessment tool used in wellbaby clinics for early detection of developmental problems, based on the developmental scales of Gesell and adapted by Touwen and Hempel) was used. ${ }^{9}{ }^{10}$ For calculation of predictive values of the brain structure T1-weighted SI comparisons for outcome, outcome groups 1 and 2 were considered to be favorable, and outcome group 3 and death, to be adverse. Neonatal clinical parameters (HIE grades according to Sarnat and Sarnat ${ }^{7}$ ) were also related to outcome.

\section{Statistical Analysis}

All statistical analysis was performed in consensus by L.L. and A.A.v.d.B.-H. All statistical evaluations were performed with the Statistical Package for the Social Sciences software, Version 11.5 (SPSS, Chicago, Ill).

Logistic regression, by using a forward stepwise selection procedure (likelihood ratio test), was performed to assess which pair-wise T1-weighted SI comparisons of the 19 brain structures were most useful to distinguish between children with normal outcome (group 1) and the 2 other outcome groups or death and between children with and without adverse outcome. The predictive value of this brain structure T1-weighted SI comparison was calculated for outcome. We also calculated predictive values of the neonatal clinical classification (controls and HIE grades 2 and 3 according to Sarnat and Sarnat ${ }^{7}$ ) for outcome. Thereafter, we separately calculated outcome prediction only in infants with HIE grade 2, because outcome of these patients is reported to be very variable. ${ }^{11-13}$ Subsequently, we evaluated whether brain structure T1-weighted SI comparison contributed to outcome prediction in infants with HIE grade 2.

\section{Results}

\section{Clinical Data}

Outcome data were available for all 57 children. Six children died in the neonatal period. There were no deaths in the postneonatal period. The normal outcome category (group 1) comprised 31 children; the mildly abnormal category (group 2), 14; and the definitely abnormal category (group 3), 6 children. Of the 34 controls, there were 30 in the normal outcome category and 4 in the mildly abnormal category at 5 years of age. The latter 4 children were subsequently classified into the group 2 category (thus did not have a normal outcome). In the HIE grade 2 group, 3 infants died, and there were 1 normal, 10 mildly abnormal, and 6 definitely abnormal outcome children. The only patient in the HIE grade 2 group with an unexpected outcome was classified to normal outcome (group 1) at 5 years of age. The HIE grade 3 group consisted of 3 patients, all of whom died in the neonatal period.

\section{Imaging Data}

The neonatal MR imaging examinations of the control infants did not show HI brain injury, and brain maturation was normal compared with normal developmental tables. ${ }^{6}$ 
Table 1: Sensitivity, specificity, and predictive value of the neonatal clinical classification for outcome

\begin{tabular}{lcccr}
\hline Outcome & Sensitivity & Specificity & PPV & NPV \\
\hline Abnormal & $85 \%$ & $97 \%$ & $96 \%$ & $88 \%$ \\
Adverse & $100 \%$ & $76 \%$ & $52 \%$ & $100 \%$ \\
\hline
\end{tabular}

Note:-PPV indicates positive predictive value; NPV, negative predictive value.

Table 2: Sensitivity, specificity, and predictive value of the brain structure T1-weighted comparison of the SI of the posterolateral putamen equal to or greater than that of the posterior limb of the internal capsule for outcome

\begin{tabular}{lcccc}
\hline Outcome & Sensitivity & Specificity & PPV & NPV \\
\hline Abnormal & $54 \%$ & $94 \%$ & $88 \%$ & $71 \%$ \\
Adverse & $92 \%$ & $89 \%$ & $69 \%$ & $98 \%$ \\
\hline
\end{tabular}

Note:-PPV indicates positive predictive value; NPV, negative predictive value. SI, signal intensity.

Table 3: Sensitivity, specificity, and predictive value of the brain structure T1-weighted comparison of the SI of the posterolateral putamen less than that of the posterior limb of the internal capsule for outcome

\begin{tabular}{lcccc}
\hline Outcome & Sensitivity & Specificity & PPV & NPV \\
\hline Abnormal & $46 \%$ & $6 \%$ & $29 \%$ & $13 \%$ \\
Adverse & $8 \%$ & $11 \%$ & $2 \%$ & $31 \%$
\end{tabular}

Note:-PPV indicates positive predictive value; NPV, negative predictive value; SI, signal intensity.

\section{Prediction of Outcome}

Neonatal Clinical Classification. First the sensitivity, specificity, and predictive values of the neonatal clinical classifications (controls or HIE grades 2 or 3 ) for outcome ( 1 of the outcome groups at 5 years of age or death) were calculated. This was done for normal outcome (outcome group 1) versus abnormal outcome (outcome groups 2 and 3 and death) and for favorable outcome (outcome groups 1 and 2) versus adverse outcome (outcome group 3 and death). Results are shown in Table 1.

Brain Structure T1-Weighted SI Comparisons. Of all pair-wise T1-weighted SI comparisons of the 19 brain structures, SI in the posterolateral putamen equal to or greater than that in the posterior limb of the internal capsule was optimal (likelihood ratio test $=37.05, P=.001$ ) for predicting normal outcome (group 1) versus abnormal outcome (outcome groups 2 and 3 and death). Sensitivity, specificity, positive predictive value, and negative predictive value of this brain structure T1-weighted SI comparison for abnormal and adverse outcome were calculated (Table 2). Sensitivity, specificity, positive predictive values, and negative predictive values of the comparison of SI in the posterolateral putamen less than that in the posterior limb of the internal capsule for abnormal and adverse outcomes were also calculated (Table 3 ).

Predictive Values of Neonatal Clinical Classification and Brain Structure T1-Weighted SI Comparisons. For abnormal outcome, the calculations of the brain structure T1weighted SI comparison were lower than the values of the neonatal clinical classification (Tables 1-3). For adverse outcome, sensitivity and negative predictive values of the brain structures T1-weighted SI comparison were lower than those of the neonatal clinical classification.

HIE Grade 2. Because the neurologic outcome of infants with HIE grade 2 is reported to be highly variable, ${ }^{11-13}$ we
Table 4: Predictive value of the neonatal clinical classification and the brain structure T1-weighted SI comparisons for adverse outcome in HIE grade 2 infants only

\begin{tabular}{llr}
\hline & PPV & NPV \\
\hline Neonatal classification & $45 \%$ & $0 \%$ \\
SI PP $\geq$ PLIC & $67 \%$ & $88 \%$ \\
SI PP $<$ PLIC & $88 \%$ & $67 \%$ \\
\hline
\end{tabular}

Note:-PPV indicates positive predictive value; NPV, negative predictive value; $\mathrm{PP}$ posterolateral putamen; PLIC, posterior limb of the internal capsule; SI, signal intensity.

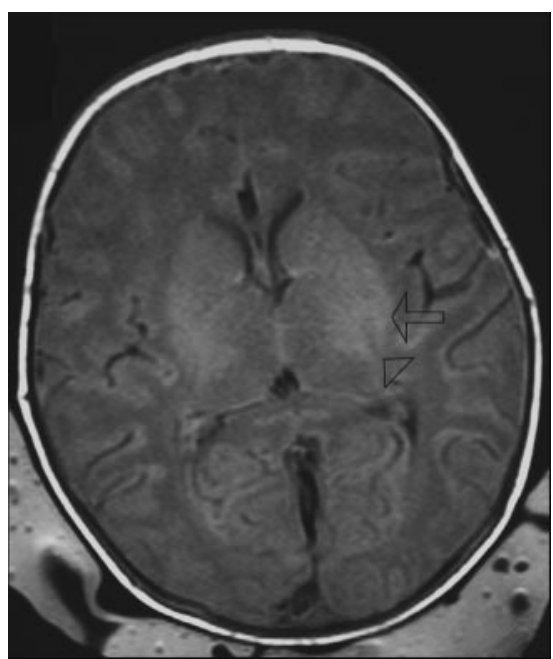

Fig 1. T1-weighted image (TR/TE, 550/14; signals acquired, 2; matrix, 205/256; section thickness, $5 \mathrm{~mm}$; section gap, $0.5 \mathrm{~mm}$; FOV, $16 \mathrm{~cm}$ ) of a neonate from the HIE group (HIE grade 2) imaged at 3 days of age. The image shows higher $\mathrm{SI}$ in the posterolateral putamen (arrow) than in the posterior limb of the internal capsule (arrowhead) and abnormal SI in the lateral thalami. The neonate was born at a gestational age of $40+4$ weeks, the mother underwent an emergency cesarean delivery for fetal distress, and the neonate was resuscitated. The Apgar score was $0-4-5$ (at 1, 5, and 10 minutes). At 5 years of age, this child has an abnormal development (outcome group 3 ).

separately calculated outcome prediction in infants with HIE grade 2 only. Outcomes of the 20 patients with HIE grade 2 at baseline were, as anticipated, quite variable: 11 had favorable outcome (outcome groups 1-2); and 9, adverse outcome (outcome group 3 or death), resulting in a positive predictive value of the neonatal clinical classification for prediction of adverse outcome of $45 \%$ (and a negative predictive value of $0 \%$ ) (Table 4).

The only child with normal outcome (group 1) had SI of the posterolateral putamen equal to that in the posterior limb of the internal capsule. In outcome group two, 3 children had SI of the posterolateral putamen equal to or greater than that of the posterior limb of the internal capsule and 7 had SI of the posterolateral putamen less than that in the posterior limb of the internal capsule. In outcome group 3, all 6 children had SI of the posterolateral putamen equal to or greater than that in the posterior limb of the internal capsule (Fig 1). Of the children who died, 2 had SI of the posterolateral putamen equal to or greater than that in the posterior limb of the internal capsule and 1 had SI of the posterolateral putamen less than that in the posterior limb of the internal capsule. Thus, of the 20 children with HIE grade two, 12 children demonstrated SI of the posterolateral putamen equal to or greater than that in the posterior limb of the internal capsule. Eight of the 12 children had adverse outcomes. This resulted in a positive predictive value for the prediction of adverse outcome of $67 \%$ and a 


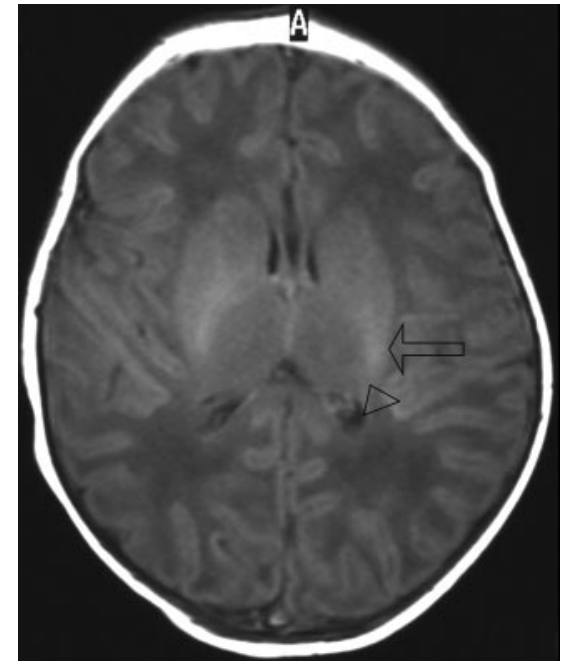

Fig 2. T1-weighted image (TR/TE, 550/14; signals acquired, 2; matrix, 205/256; section thickness, $5 \mathrm{~mm}$; section gap, $0.5 \mathrm{~mm}$; FOV, $16 \mathrm{~cm}$ ) of a neonate from the control group imaged at 2 days of age. The image shows higher $\mathrm{SI}$ in the posterior limb of the internal capsule (arrowhead) than in the posterolateral putamen (arrow). The child was a term-born neonate with a simple lumbar meningocele. At the age of 5 years, this child has a normal development (outcome group 1).

negative predictive value of $88 \%$ (Table 4). Four of these 12 neonates with SI of the posterolateral putamen equal to or greater than that in the posterior limb of the internal capsule had favorable outcomes ( 1 outcome group 1, 3 outcome group 2 ). The child with normal outcome (group 1) was born asphyxiated due to maternal shock and a complicated delivery. The Apgar score was 0-5-7 (at 1, 5, and 10 minutes). MR imaging performed on the fourth day after birth showed no abnormalities besides subdural effusions. SI in the posterolateral putamen was equal to the SI in the posterior limb of the internal capsule.

Of the 20 patients with HIE grade two, 8 demonstrated SI of the posterolateral putamen less than that of the posterior limb of the internal capsule (Fig 2), of whom only 1 had an adverse outcome, resulting in a positive predictive value for prediction of adverse outcome of $88 \%$ and a negative predictive value of $67 \%$ (Table 4). The only child with an SI of the posterolateral putamen less than that of the posterior limb of the internal capsule with adverse outcome (neonatal death) was born after a gestational age of 41 weeks by emergency cesarean delivery because of breech delivery. The Apgar score was 0-5-5 (at 1, 5, and 10 minutes). He developed meconium aspiration syndrome. MR imaging performed on the third day after birth showed diffuse edema but no abnormal SI in the basal ganglia. The infant died 7 days after birth.

Thus, in the HIE grade 2 group, neonatal MR imaging with comparison of SI between the posterolateral putamen and the posterior limb of the internal capsule proved to be of added value (positive predictive value for prediction of adverse outcome of $67 \%$ and a negative predictive value of $88 \%$ ) to the neonatal clinical classification (positive predictive value for prediction of adverse outcome of $45 \%$ and a negative predictive value of $0 \%$ ) (Table 4 ).

\section{Discussion}

We previously demonstrated that SI comparisons of brain structures on T1-weighted images were valuable in predicting the absence or presence of HI brain injury. The aim of the present study was to assess the value of this method in predicting developmental outcome. We systematically studied SI comparisons of several brain structures on T1-weighted imaging and related them to outcome. For surviving infants, our rationale to assess developmental outcome at 5 years of age was twofold: First, at this age, children need to perform more specialized tasks than preschool children; and second, in younger children, outcome may still change with time. Mild motor problems may be overlooked at a younger age, and mental delay may remain undetected before school age. Minor abnormalities, such as clumsiness or mild delay, are better recognized at school age than in younger children. ${ }^{2}$ On the other hand, mild motor problems at a young age may still improve. $^{2,11}$

We found that the T1-weighted SI comparison of the posterolateral putamen versus the posterior limb of the internal capsule resulted in the best prediction of normal or abnormal outcome and of favorable or adverse outcome and resulted in the optimal prediction of outcome. We previously found that the T1-weighted SI comparison of the posterolateral putamen versus the posterior limb of the internal capsule was also the optimal comparison to distinguish infants with HIE from control subjects. ${ }^{7}$

Our sensitivity for adverse outcome is comparable with the results of Rutherford et al, ${ }^{14}$ who studied whether abnormal SI of the posterior limb of the internal capsule on MR imaging predicted outcome at 1 year of age in infants with HIE. We obtained a higher negative predictive value (98\%) (Table 2), whereas they obtained higher values of specificity and positive predictive value. ${ }^{14}$ However, Rutherford et al related SI in the posterior limb of the internal capsule to development at the age of 1 year. Our predictive values are comparable with those of other authors who related MR spectroscopy findings to clinical outcome. ${ }^{15,16}$ However, their period of clinical follow-up was much shorter, a maximum of 24 months. Kadri et $\mathrm{al}^{17}$ combined clinical, MR spectroscopy, and EEG findings and found a sensitivity of $86 \%$ and a negative predictive value of $88 \%$ for the combination of MR spectroscopy/HIE score/ EEG. They related this to neurologic outcome at 2 years of age.

Van Schie et $\mathrm{al}^{5}$ related MR imaging findings in full-term neonates with HIE to motor ability and neurologic performance at 1 year of age. For MR imaging, they used the scoring system for basal ganglia/watershed scores as proposed by Barkovich et $\mathrm{al}^{18}$; abnormal SI in the basal ganglia, thalamus, or cortex was scored. Also, special attention was paid to the posterior limb of the internal capsule. MR imaging was performed at a median age of 7 days (range, 1-45 days). The MR imaging findings did not predict early motor outcome. However, sensitivity, specificity, and predictive values were not calculated, and outcome was only evaluated at 1 year of age.

El-Ayouty et al $^{19}$ studied the relation between EEG and MR imaging findings in full-term neonates with HIE and calculated the predictive values of EEG background activity and imaging findings for outcome at 1 1/2 years of age. On MR imaging, they assessed abnormal SI of the posterior limb of the internal capsule, basal ganglia, thalami, and white matter (subcortical, periventricular, or widespread). MR imaging was performed at a median age of 15 days (range, 8-25 days). Sensitivity for poor outcome of abnormal MR examinations 
was $100 \%$; specificity, $43 \%$; positive predictive value, $82 \%$; and negative predictive value, $100 \%$. These findings mean that except for specificity, their values were higher than those obtained in our study. However, outcome was only evaluated to 18 months of age.

Leijser et $\mathrm{al}^{20}$ also studied prediction of short-term outcome in (near) term neonates with HIE, combining neuroimaging (cranial ultrasonography and MR imaging) and EEG. On MR imaging, SI of the deep gray matter, posterior limb of the internal capsule, white matter, and cortex was assessed. MR imaging was performed 1-7 days after birth with a mean age of 4 days. Sensitivity, specificity, positive predictive value, and negative predictive value of normal to mildly abnormal MR imaging findings for prediction of favorable outcome were $38 \%, 93 \%, 75 \%$, and 74\%, respectively. Severely abnormal MR imaging findings predicted unfavorable outcome. Values were respectively $74 \%, 100 \%, 100 \%$, and $67 \%$, thus higher specificity and positive predictive value but lower negative predictive value than those in our brain structure T1weighted SI comparison.

In the present study, the neonatal clinical classification provided the best predictive values, except for the positive predictive value for adverse outcome (Tables 1-3). We classified young infants on the basis of clinical parameters in the early neonatal period into 3 groups (controls, patients with HIE grade 2 , and patients with HIE grade 3 according to Sarnat and Sarnat ${ }^{7}$ ). Except in patients with HIE grade 2, this classification corresponded well with outcome at 5 years of age (Table 1). Control infants generally had a normal outcome. The 3 infants with HIE grade 3 died in the neonatal period. As many as 95\% (19/20) of infants classified with HIE grade 2 group had an abnormal outcome. Their outcome, however, varied from normal to death. Outcome of infants with HIE grade 2 is highly variable. ${ }^{11-13}$ The predictive values of the neonatal classification for outcome prediction in the subgroup of children with HIE grade 2 were low. Using the brain structure T1weighted SI comparison in the posterolateral putamen and the posterior limb of the internal capsule for prediction of outcome in these children, the predictive values were higher as compared with the predictive values of the neonatal clinical classification only. For clinicians, patients with HIE grade 2 are a very important patient group because their outcome is so highly variable, difficult to predict, and possibly unfavorable.

In MR imaging patterns of HI brain injury, white matter atrophy, the presence of abnormal SI in the basal ganglia and/or thalamus, or abnormal SI on T1-weighted images in the posterior limb of the internal capsule have been shown to be predictive for poor outcome..$^{5,8,18,21-23}$ Yet only a few authors have calculated the sensitivity and specificity for outcome prediction. ${ }^{14,16,19,20}$ Rutherford et al ${ }^{14}$ found high predictive values and high sensitivity and specificity for abnormal or equivocal SI in the posterior limb of the internal capsule, as discussed previously. However, their follow-up was reported only up to 12 months. ${ }^{14}$ Jyoti et al ${ }^{16}$ obtained positive predictive values for normal/near-normal outcome and poor outcome on the basis of a combination of a cerebral palsy score and a Griffiths Mental Development Assessment at 1 year of age. However, neither sensitivity and specificity nor negative predictive values were reported. ${ }^{16}$

In our previous and present studies, we included all chil- dren (neonates with HIE stage 2 lasting at least 5 days or HIE stage 3 according to Sarnat and Sarnat ${ }^{7}$ ) who were consecutively admitted to our neonatal unit. This clinical setting comes with several limitations. First of all, there is a variability of the MR imaging acquisition parameters used. Different MR imaging protocols (different TRs and section thicknesses) were used for children with various ages, which might slightly influence the relative SI of the individual brain structures. Moreover, the clinical setting also resulted in our control group not consisting of healthy infants. Of the 34 control infants, 30 had a normal outcome. Four were classified in outcome group two, 2 of whom had neural tube defects. Patients with neural tube defects are reported to have a higher incidence of cognitive impairment. ${ }^{24}$ Brain maturation of our infants with neural tube defects appeared normal compared with normal developmental tables. Omitting these children from our calculations would probably have improved our results. However, this would not give a correct impression of the actual clinical situation. Also, the children in outcome group 4 were highly selected because withdrawal of treatment occurred in infants with HIE grade 3 who had abnormal neuroimaging findings and severely depressed background activity on EEG. A last drawback of our study is that we have not as yet prospectively analyzed the reliability and reproducibility of this brain structure T1-weighted SI comparison method, which could possibly be of influence on the present results.

\section{Conclusion}

Brain structure T1-weighted SI comparisons are helpful to predict outcome in (near) term neonates with HIE. These add to the current knowledge and clinical practice but would need to be validated on a larger scale. If SI in the posterolateral putamen is less than that in the posterior limb of the internal capsule, favorable outcome is very likely in neonates with HIE. However, if SI of the posterolateral putamen is equal to or greater than that of the posterior limb of the internal capsule, adverse outcome is very likely. In neonates with HIE grade 2 according to Sarnat and Sarnat, a patient group with highly variable outcome, prediction of outcome is substantially improved by using these brain structure T1-weighted SI comparisons.

\section{Acknowledgment}

We thank Y.L. Hoogeveen, PhD, of the Department of Radiology, Radboud University Nijmegen Medical Center, for her help with manuscript preparation.

\section{References}

1. Mercuri E, Barnett A, Rutherford M, et al. Neonatal cerebral infarction and neuromotor outcome at school age. Pediatrics 2004;113:95-100

2. Barnett A, Mercuri E, Rutherford M, et al. Neurological and perceptual-motor outcome at 5-6 years of age in children with neonatal encephalopathy: relationship with neonatal brain MRI. Neuropediatrics 2002;33:242-48

3. Mercuri E, Barnett AL. Neonatal brain MRI and motor outcome at school age in children with neonatal encephalopathy: a review of personal experience. Neural Plast 2003;10:51-57

4. Krageloh-Mann I, Helber A, Mader I, et al. Bilateral lesions of thalamus and basal ganglia: origin and outcome. Dev Med Child Neurol 2002;44:477-84

5. van Schie PE, Becher JG, Dallmeijer AJ, et al. Motor outcome at the age of one after perinatal hypoxic-ischemic encephalopathy. Neuropediatrics 2007;38:71-77

6. Liauw L, Palm-Meinders IH, van der Grond J, et al. Differentiating normal myelination from hypoxic-ischemic encephalopathy on T1-weighted MR images: A new approach. AJNR Am J Neuroradiol 2007;28:660-65 
7. Sarnat HB, Sarnat MS. Neonatal encephalopathy following fetal distress: a clinical and electroencephalographic study. Arch Neurol 1976;33:696-705

8. Boichot C, Walker PM, Durand C, et al. Term neonate prognoses after perinatal asphyxia: contributions of MR imaging, MR spectroscopy, relaxation times, and apparent diffusion coefficients. Radiology 2006;239:839-48

9. Gesell A, Amatruda CS. Developmental diagnosis: normal and abnormal child development. In: Knobloch H, Pasamanick B, Hagerstown MD, eds. Developmental Diagnosis: The Evaluation and Management of Normal and Abnormal Neuropsychologic Development in Infancy and Early Childhood. 3rd ed. New York: Harper and Row; 1974

10. Brouwers-de Jong EA, Burgmeijer RJF, Laurent de Angulo MS. Ontwikkelingsonderzoek op het consultatiebureau. In: Handboek bij het vernieuwde Van Wiechenonderzoek. Assen, the Netherlands: Van Gorcum; 1996

11. Cowan F. Outcome after intrapartum asphyxia in term infants. Semin Neonatol 2000;5:127-40

12. Dilenge ME, Majnemer A, Shevell MI. Long-term developmental outcome of asphyxiated term neonates. J Child Neurol 2001;16:781-92

13. Haataja L, Mercuri E, Guzzetta A, et al. Neurologic examination in infants with hypoxic-ischemic encephalopathy at age 9 to 14 months: use of optimality scores and correlation with magnetic resonance imaging findings. J Pediatr 2001;138:332-37

14. Rutherford MA, Pennock JM, Counsell SJ, et al. Abnormal magnetic resonance signal in the internal capsule predicts poor neurodevelopmental outcome in infants with hypoxic-ischemic encephalopathy. Pediatrics 1998;102:323-28

15. Hanrahan JD, Cox IJ, Azzopardi D, et al. Relation between proton magnetic resonance spectroscopy within 18 hours of birth asphyxia and neurodevelopment at 1 year of age. Dev Med Child Neurol 1999;41:76-82
16. Jyoti R, O’Neil R. Predicting outcome in term neonates with hypoxic-ischaemic encephalopathy using simplified MR criteria. Pediatr Radiol 2006;36:38-42

17. Kadri M, Shu S, Holshouser B, et al. Proton magnetic resonance spectroscopy improves outcome prediction in perinatal CNS insults. J Perinatol 2003;23:181-85

18. Barkovich AJ, Hajnal BL, Vigneron D, et al. Prediction of neuromotor outcome in perinatal asphyxia: evaluation of MR scoring systems. AJNR Am J Neuroradiol 1998;19:143-49

19. El-Ayouty M, Abdel-Hady H, El-Mogy S, et al. Relationship between electroencephalography and magnetic resonance imaging findings after hypoxicischemic encephalopathy at term. Am J Perinatol 2007;24:467-73

20. Leijser LM, Vein AA, Liauw L, et al. Prediction of short-term neurological outcome in full-term neonates with hypoxic-ischaemic encephalopathy based on combined use of electroencephalogram and neuro-imaging. Neuropediatrics 2007;38:219-27

21. Rutherford M, Pennock J, Schwieso J, et al. Hypoxic-ischaemic encephalopathy: early and late magnetic resonance imaging findings in relation to outcome. Arch Dis Child Fetal Neonatal Ed 1996;75:F145-51

22. de Vries LS, van der Grond J, van Haastert IC, et al. Prediction of outcome in new-born infants with arterial ischaemic stroke using diffusion-weighted magnetic resonance imaging. Neuropediatrics 2005;36:12-20

23. Hunt RW, Neil JJ, Coleman LT, et al. Apparent diffusion coefficient in the posterior limb of the internal capsule predicts outcome after perinatal asphyxia. Pediatrics 2004;114:999-1003

24. Vinck A, Maassen B, Mullaart R, et al. Arnold-Chiari-II malformation and cognitive functioning in spina bifida. J Neurol Neurosurg Psychiatry 2006;77: 1083-86. Epub 2006 May 11 